\title{
Synthesis and Biological Evaluation of Methylenecyclopropane Analogues of Nucleosides
}

\author{
Germain Obame, ${ }^{\mathrm{a}}$ Paul Brémond, ${ }^{\mathrm{a}}$ Christophe Pannecouque, ${ }^{\mathrm{b}}$ Gérard Audran*a \\ a Aix-Marseille Université, CNRS ICR - UMR 7273, 13397 Marseille Cedex 20, France \\ b Rega Institute for Medical Research, Katholieke Universiteit Leuven, 10 Minderbroedersstraat, 3000 Leuven, Belgium \\ Fax +33(4)91288545; E-mail: g.audran@univ-amu.fr
}

Received: 13.05.2013; Accepted after revision: 04.06.2013

\begin{abstract}
Starting from a chiral methylenecyclopropane building block, readily obtained by enzymatic desymmetrization of a mesodiol, two types of methylenecyclopropane analogues of nucleosides were synthesized. The first type of nucleosides was obtained from the direct coupling of the chiral building block with 6-chloropurine under Mitsunobu reaction conditions followed by the functionalization of the purine base. The second type of nucleosides featured a Curtius rearrangement as the key step and the uracil heterocycle was then constructed by a linear methodology. These derivatives were evaluated as potential agents against important viral pathogens. None of the new compounds had significant antiviral activity at a concentration of $100 \mu \mathrm{g} / \mathrm{mL}$, which was the highest concentration tested.
\end{abstract}

Key words: stereoselective synthesis, Curtius rearrangement, carbocyclic nucleosides, methylenecyclopropane carbonucleosides, antiviral agents

Nucleoside analogues represent an important class of therapeutic agents and show a remarkable wide spectrum of antiviral chemotherapy. ${ }^{1}$ Among them, carbocyclic nucleosides are nucleoside analogues in which a carbon atom has replaced the oxygen atom of the furanose ring. They have been extensively studied including carbovir, ${ }^{2}$ abacavir ${ }^{3}\left(\right.$ Ziagen $\left.^{\circledR}\right)$, and some cyclopentanic nucleosides. ${ }^{4}$ In fact, they can display similar biological activity while presenting better stability as they are not prone to the action of the glycosylases that cleave the glycosidic bond of the natural nucleosides. As an example, entecavir ${ }^{5}$ (Baraclude ${ }^{\circledR}$ ), a carbanucleoside possessing a methylenecyclopentane skeleton, has been approved for the treatment of HBV infection (Figure 1). Moreover, in the course of the synthesis of novel antiviral nucleoside analogues, structural modifications of the heterocyclic bases and/or modifications on the sugar moiety have been attempted. ${ }^{6}$ As a consequence, novel nucleosides containing a cyclopropane moiety have been found to have potent antiviral activity. Thus, methylenecyclopropane analogues of nucleosides, ${ }^{7}$ such as synadenol ${ }^{8}$ and cyclopropavir, ${ }^{9}$ have been established as antiviral agents against $\beta$-herpes viruses and $\gamma$-herpes viruses. ${ }^{10}$ In this paper, we report the synthesis and biological evaluation, with full experimental details, of novel methylenecyclopropane analogues of nucleosides.

SYNTHESIS 2013, 45, 2612-2618

Advanced online publication: 12.07.2013

DOI: 10.1055/s-0033-1339311; Art ID: SS-2013-T0204-OP

(C) Georg Thieme Verlag Stuttgart $\cdot$ New York

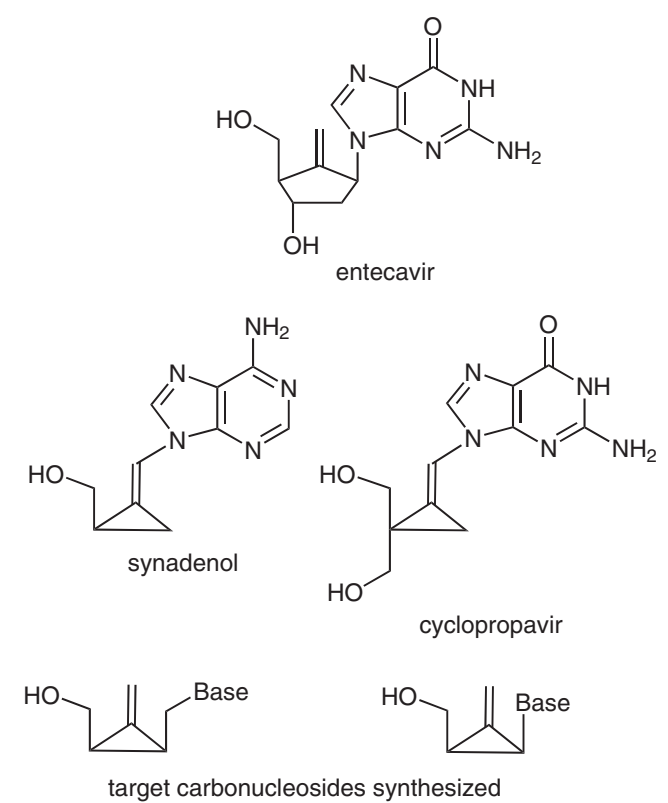

Figure 1 The structures of entecavir (Baraclude ${ }^{\circledR}$ ), synadenol, cyclopropavir, and the methylenecyclopropane nucleosides synthesized in this work

We recently published a short paper on the synthesis and the enzymatic desymmetrization of meso-diol ${ }^{11} \mathbf{1}$ (Scheme 1) that is used herein for the synthesis of novel types of methylenecyclopropane nucleosides. The synthesis of meso-diol 1 was accomplished in $64 \%$ yield in three steps starting from 2,2-dimethyl-4,7-dihydro-1,3-dioxepin, which is easily prepared according to a literature procedure. ${ }^{12}$ First, addition of methylchlorocarbene, ${ }^{13}$ generated in situ from 1,1-dichloroethane and butyllithium in hexanes at $-35{ }^{\circ} \mathrm{C}$, to the dioxepin gave a $4: 1$ (by ${ }^{1} \mathrm{H}$ NMR) trans/cis mixture of the expected diastereomeric chlorocyclopropanes. Then, dehydrochlorination of the chlorocyclopropanes with potassium tert-butoxide in dimethyl sulfoxide afforded methylenecyclopropane 2 in $76 \%$ yield, which was hydrolyzed with $2 \mathrm{M}$ hydrochloric acid to give the diol 1 in $81 \%$ yield.

After screening commercial lipases, (-)-4 was obtained on a large scale in $86 \%$ yield and an enantioselectivity of 98\% ee by using lipase AK (from Pseudomonas fluorescens, PFL) and vinyl acetate in diisopropyl ether (Scheme $2)$. The high enantiomeric purity of (-)-4 was determined by HPLC (Figure 2). Moreover, the stereostructure of $\mathbf{4}$ 


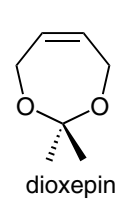<smiles>[Y]C1(C)OCC2C(=C)C2CO1</smiles><smiles>C=C(CO)[C@H](O)CO</smiles>

Scheme 1

was secured by single crystal X-ray diffraction of the corresponding camphanate ester derivative used. As depicted in Scheme 2, the reaction of (-)-4 with $(1 S, 4 R)$-camphanic chloride $^{14}$ afforded the derivative (-)-5 in $91 \%$ yield, which was obtained as an oil. To solve this problem we performed the selective hydrolysis of the acetate group. Thus, treatment of (-)-5 with potassium carbonate in methanol afforded the crystalline alcohol $(+)-6$ in $86 \%$ yield. The ORTEP view of $(+)-6$ is shown in Figure $2 .{ }^{15}$ The synthesis of the enantiomer $(+)-4$, by desymmetrization of diacetate $\mathbf{3}$, was also accomplished. Thus, treatment of 1 with acetic anhydride and pyridine gave the meso-diacetate 3 in 91\% yield. Treatment of 3 with PFL in the presence of butanol in diisopropyl ether afforded $(+)-4$ in $82 \%$ yield and with $>98 \%$ ee. The high enantiomeric purity of (+)-4 was also checked by HPLC (Figure 2 ). This methodology provides a very simple and practical tool to access the two enantiomers of a versatile building block used to synthesize the target nucleosides, both on a large scale and with high enantiomeric excess.

The syntheses of methylenecyclopropane analogues of nucleosides are outlined in Scheme 3. Derivative (-)-4 was treated with 6-chloropurine in the presence of diisopropyl azodicarboxylate (DIAD) and triphenylphosphine to give the corresponding chloropurine (+)-7 in $86 \%$ yield. ${ }^{16}$ One-pot ammonolysis and deprotection of the acetyl group of $(+)-7$ by treatment with saturated methanolic ammonia solution at $100{ }^{\circ} \mathrm{C}$ in a stainless steel pressure vessel for 10 hours provided $92 \%$ yield of aminopurine (-)-8. Alternatively, treatment of $(+)-7$ with cyclopropylamine followed by cleavage of the acetyl protecting group with methanolic ammonia produced the target aminopurine (-)-9 in 89\% yield for two steps.

The methodology for the synthesis of another type of methylenecyclopropane analogue of nucleosides used the Curtius reaction ${ }^{17}$ as a key step in order to remove a carbon atom and therefore to attach the heterocyclic base di-

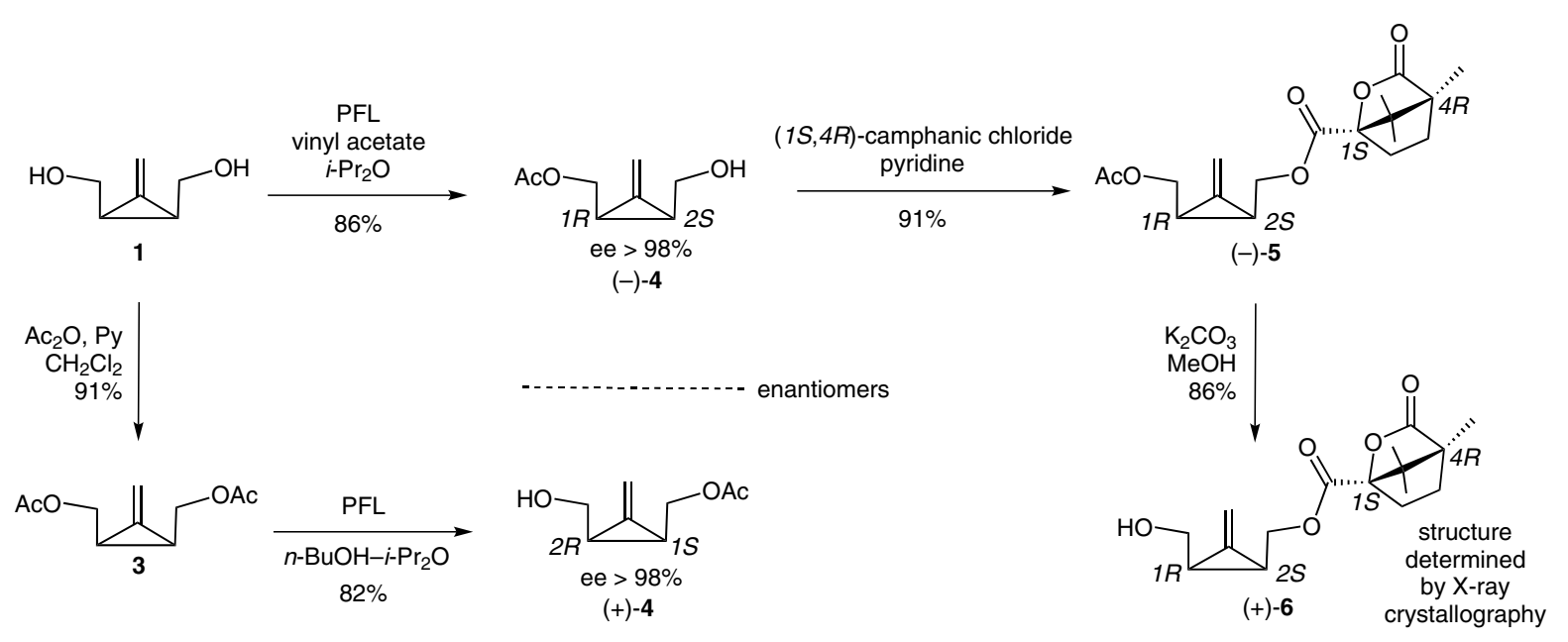

Scheme 2
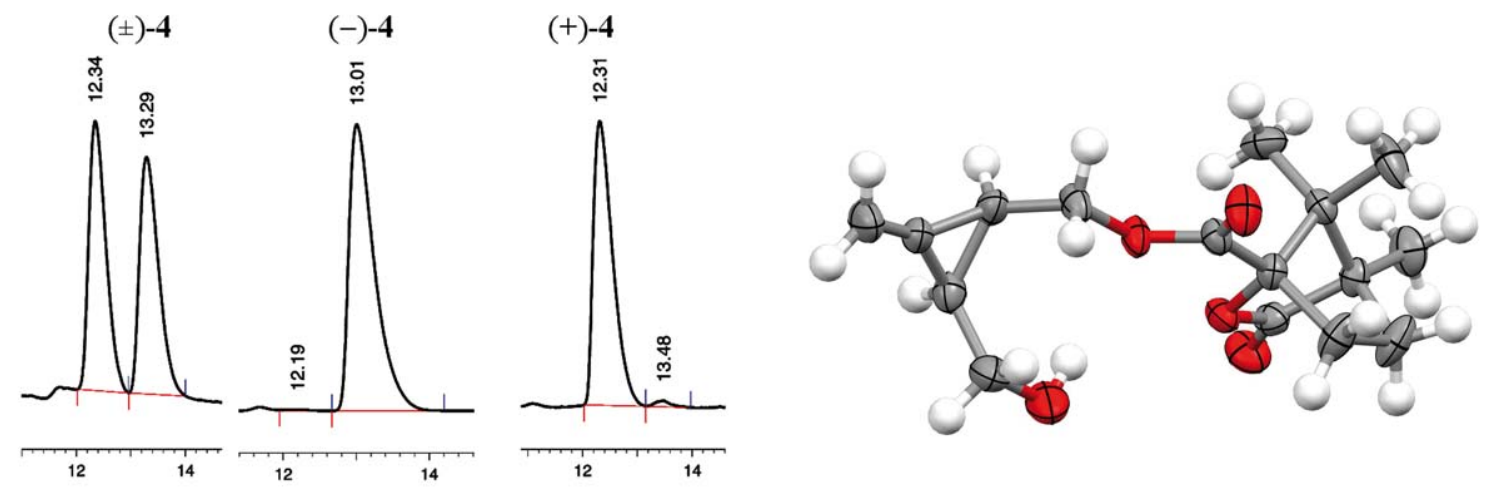

Figure 2 Chiral HPLC diagrams of ( $( \pm)-4,(-)-4$ and (+)-4 and ORTEP view of chiral camphanate derivative (+)-6 


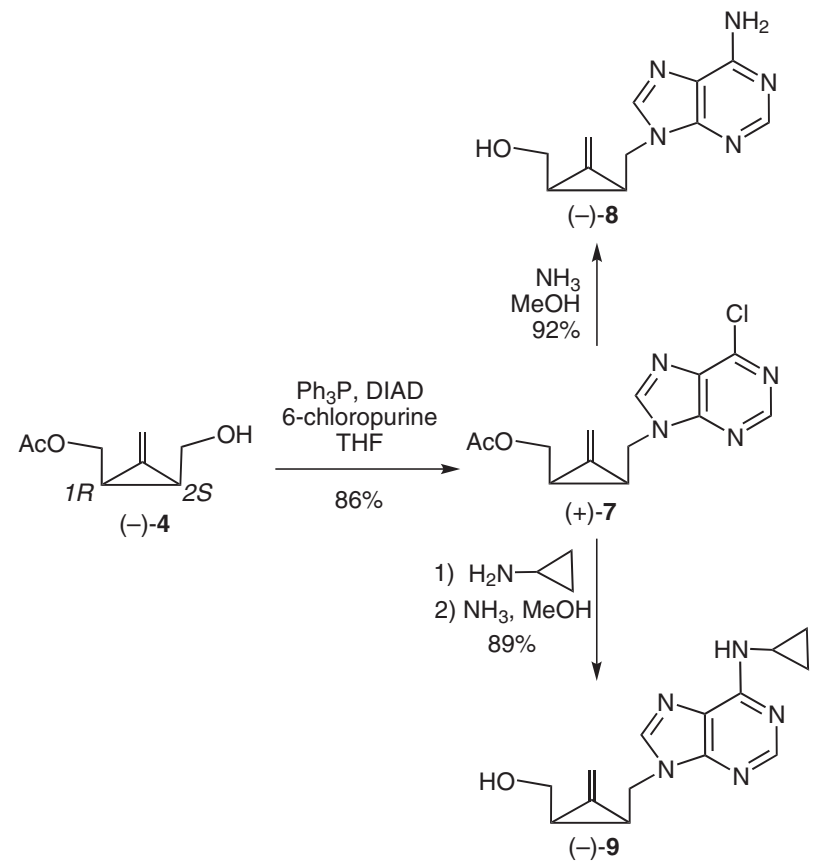

Scheme 3

rectly to the carbasugar (Scheme 4). Thus, Jones' reagent was employed to oxidize the alcohol (-)-4 in acetone at $0{ }^{\circ} \mathrm{C}$ to the corresponding carboxylic acid (-)-10 in $91 \%$ yield. ${ }^{18}$ Direct transformation of carboxylic acid to the acyl azide (+)-11 with diphenylphosphoryl azide and triethylamine in dichloromethane failed and no reaction was observed. ${ }^{19}$ The carboxylic acid (-)-10 was then converted into the acid chloride with oxalyl chloride at $0{ }^{\circ} \mathrm{C}$ and then to the acyl azide (+)-11 by using sodium azide in a water-acetone mixture. ${ }^{20}$ Then, Curtius rearrangement of acyl azide (+)-11 to the corresponding isocyanate $\mathbf{1 2}$ was conducted in benzene, but the temperature should be kept below $75^{\circ} \mathrm{C}$ as we observed decomposition of thus formed isocyanate derivative and this resulted in lower yields. As isocyanate $\mathbf{1 2}$ is unstable, the addition under neutral, acidic, and basic conditions of water, ${ }^{21}$ methanol, ${ }^{17 b}$ or butanol ${ }^{22}$ were attempted directly with the crude isocyanate 12, but all these reactions were unsuccessful and led to decomposition of the starting material.

Fortunately, addition of tert-butyl alcohol to the crude isocyanate $\mathbf{1 2}$ at room temperature, followed by heating of the reaction mixture at $75^{\circ} \mathrm{C}$ gave the carbamate $(+)-13$ in $75 \%$ yield for the two steps after purification by flash chromatography on silica gel. ${ }^{23}$ Then, the simultaneous deprotection of the carbamate and acetate functions of $(+)-\mathbf{1 3}$ was conducted in acidic conditions to afford the hydrochloride salt of amine $\mathbf{1 4}$ in $85 \%$ yield. ${ }^{19 a}$ After the completion of the synthesis of the carbocyclic aminosugar bearing the methylenecyclopropane moiety, the final stage involved the introduction of the heterocyclic base. All attempts to provide methylenecyclopropane analogues of nucleosides by construction of the purine base failed. Indeed, the condensation of $\mathbf{1 4}$ with 4,6-dichloropyrimidin-2-amine in the presence of diisopropylethylamine in butanol at reflux did not give the expected pyrimidine intermediate. ${ }^{24}$ As a consequence, we investigated the synthesis of pyrimidine heterocyclic base as the precursor needed to react with the carbosugar is much more reactive, and so the coupling reaction could be realized under mild conditions. Then, the uracil base was constructed with a previously adapted methodology. ${ }^{25}$ Ethyl vinyl ether was reacted with chlorocarbonyl isocyanate and the reaction mixture was treated with triethylamine to provide the highly reactive isocyanate intermediate. Condensation at $-40{ }^{\circ} \mathrm{C}$ of this isocyanate with the hydrochloride salt $\mathbf{1 4}$ in the presence of triethylamine gave the preformed uracil base $(+)-\mathbf{1 5}$. Finally, treatment of the $(+)-15$ with $0.5 \mathrm{M}$ sulfuric acid provided the uracil derivative (+)-16. This two-step sequence gave (+)-16 in 75\% yield from 14 after purification by column chromatography on silica gel. The enantioselective synthesis of the methylenecyclopropane (+)-16 with a uracil moiety as heterocyclic base was accomplished in eight steps in $34 \%$ overall yield from the chiral building block (-)-4.

Compounds 8, 9, and $\mathbf{1 6}$ were evaluated for their antiviral activity in human embryonic lung (HEL) cells [herpes simplex virus type 1 (HSV-1, strain KOS), herpes simplex virus type 2 (HSV-2, strain G), vaccinia virus, vesicular

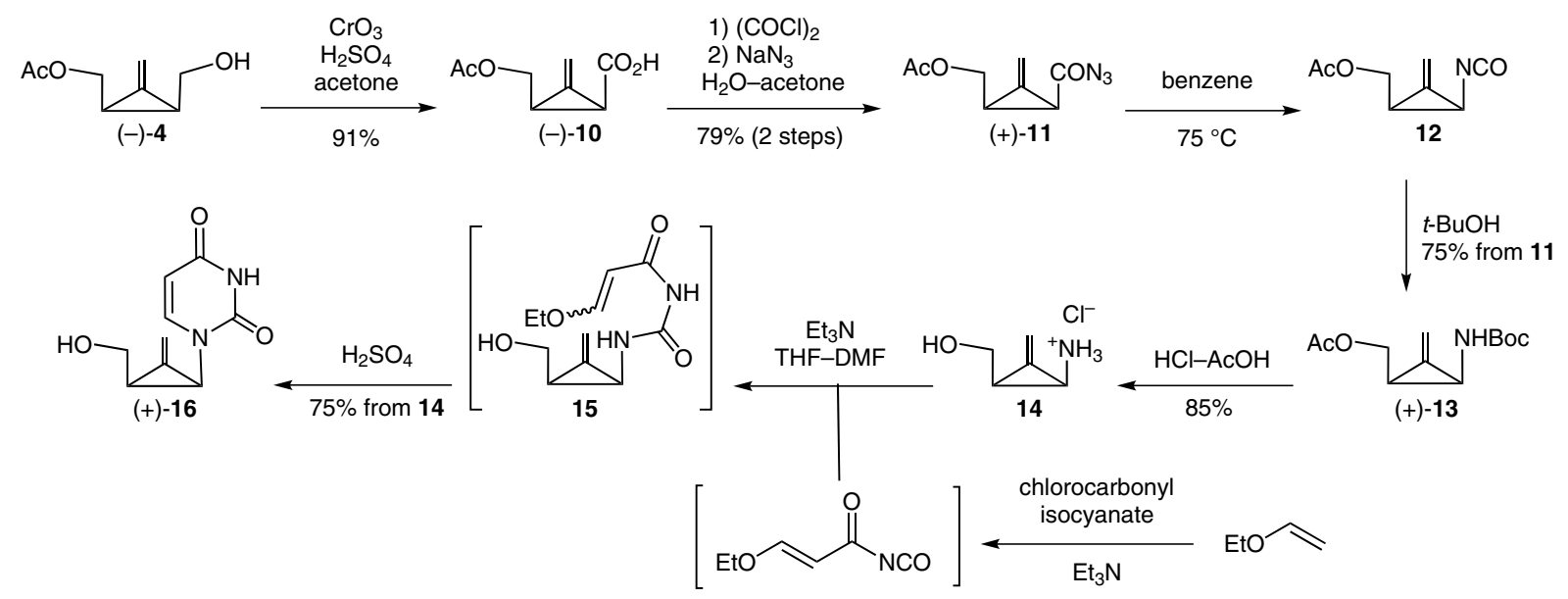

Scheme 4 
stomatitis virus, thymidine kinase-deficient (TK-) HSV-1 (strain $\mathrm{KOS}, \mathrm{ACV}^{\mathrm{r}}$ )], Vero cells (parainfluenza type 3 virus, reovirus type 1 , Sindbis virus, Coxsackie B4 virus, Punta Toro virus), HeLa cells (vesicular stomatitis virus, Coxsackie B4 virus, respiratory syncytial virus), and Madin Darby canine kidney cells (influenza A H3N2, influenza A H1N1 and influenza B). In no case was significant antiviral activity or cytotoxicity witnessed at a concentration of $100 \mu \mathrm{g} / \mathrm{mL}$, which was the highest concentration tested.

In summary, the enantioselective synthesis of methylenecyclopropane analogues of nucleosides $\mathbf{8 , 9}$, and $\mathbf{1 6}$ was achieved starting from acetate 4 obtained by desymmetrization of a meso-diol. Thus, the nucleosides were synthesized by a direct coupling of the chiral building block under Mitsunobu conditions or by Curtius rearrangement followed by a linear methodology to construct the uracil heterocycle. Synthesized compounds were tested as potential antiviral agents; none of these compounds showed antiviral activity.

All air and/or $\mathrm{H}_{2} \mathrm{O}$ sensitive reactions were performed under an argon atmosphere with dry, freshly distilled solvents using standard syringe-cannula/septa techniques; all glassware was oven-dried $\left(80^{\circ} \mathrm{C}\right)$ and/or carefully dried in line with a flameless heat gun. All solvents were distilled under an argon atmosphere: THF from a blue soln of $\mathrm{Na}$ /benzophenone ketyl radical prior to use; $\mathrm{CH}_{2} \mathrm{Cl}_{2}$ and DMF from $\mathrm{CaH}_{2}$, benzene and $\mathrm{Et}_{2} \mathrm{O}$ from $\mathrm{LiAlH}_{4}$. Routine monitoring of reactions was performed using Merck Silica gel $60 \mathrm{~F}_{254}$, aluminum-supported TLC plates; spots were visualized using UV light and ethanolic acidic $p$-anisaldehyde soln or ethanolic phosphomolybdic soln, followed by heating. Purification by column chromatography was performed with silica gel 60 (230-400 mesh) and gradients of $\mathrm{Et}_{2} \mathrm{O}$-petroleum ether or $\mathrm{CH}_{2} \mathrm{Cl}_{2}-\mathrm{MeOH}$, unless otherwise stated. ${ }^{1} \mathrm{H}$ and ${ }^{13} \mathrm{C}$ NMR spectra were recorded in $\mathrm{CDCl}_{3}$, $\mathrm{MeOH}-d_{4}$, or DMSO- $d_{6}$ on a Bruker Avance DPX-300 spectrometer; residual nondeuterated solvent was used as internal reference. Optical rotations were measured on a Perkin-Elmer 241 polarimeter. Melting points are uncorrected. HRMS were performed using a SYNAPT G2 HDMS (Waters) mass spectrometer equipped with a pneumatically assisted atmospheric pressure ionization. The sample was ionized in positive mode electrospray under the following conditions: electrospray voltage (ISV): $2800 \mathrm{~V}$; orifice voltage (OR): $20 \mathrm{~V}$; nebulizing gas flow $\left(\mathrm{N}_{2}\right): 800 \mathrm{~L} / \mathrm{h}$. The mass spectrum was obtained using a time of flight analyzer (TOF). The measure was realized in triplicate, with double internal standardization. The sample was dissolved in $\mathrm{CH}_{2} \mathrm{Cl}_{2}(450 \mu \mathrm{L})$ then diluted (dilution factor $\left.1 / 10^{3}\right)$ in a methanolic soln of $\mathrm{NH}_{4} \mathrm{OAc}(3 \mathrm{mM})$. The sample soln was infused in the ionization source at a $10 \mu \mathrm{L} / \mathrm{min}$ flow rate. Lipase AK (from Pseudomonas fluorescens, PFL) was gifted by Amano Pharmaceutical (Nagoya, Japan).

4,4-Dimethyl-8-methylene-3,5-dioxabicyclo[5.1.0]octane (2) A soln of 2,2-dimethyl-4,7-dihydro-1,3-dioxepin (20.0 g, 156 $\mathrm{mmol})$ and 1,1-dichloroethane $(13.0 \mathrm{~mL}, 156 \mathrm{mmol})$ in anhyd $\mathrm{Et}_{2} \mathrm{O}$ $(100 \mathrm{~mL})$ was cooled at $-35^{\circ} \mathrm{C}$. Then, $2.5 \mathrm{M} \mathrm{BuLi}$ in hexane $(61.6$ $\mathrm{mL}, 156 \mathrm{mmol}$ ) was added to the mixture over a period of $3 \mathrm{~h}$, during which time the reaction temperature was maintained between -40 and $-35^{\circ} \mathrm{C}$. The mixture was stirred at $-35^{\circ} \mathrm{C}$ for $14 \mathrm{~h}$, and then it was warmed to $0{ }^{\circ} \mathrm{C}$ and quenched with $\mathrm{H}_{2} \mathrm{O}$. The aqueous layer was extracted with $\mathrm{Et}_{2} \mathrm{O}$, and the combined $\mathrm{Et}_{2} \mathrm{O}$ extracts were dried, filtered, and concentrated. The oily residue was used in the next reaction without further purification.
To this crude product $(26.7 \mathrm{~g}, 140 \mathrm{mmol})$ dissolved in anhyd DMSO $(100 \mathrm{~mL}), t$-BuOK $(15.3 \mathrm{~g}, 140 \mathrm{mmol})$ was added and the resulting mixture was stirred at $70{ }^{\circ} \mathrm{C}$ for $1 \mathrm{~h}$. The soln was then cooled to r.t., diluted with brine, followed by repeated extraction with $\mathrm{Et}_{2} \mathrm{O}$. The combined organic layers were dried (anhyd $\mathrm{MgSO}_{4}$ ), filtered, and concentrated. The crude product was purified by flash chromatography to give methylenecyclopropane $2(18.3 \mathrm{~g}, 76 \%)$ as a colorless oil.

IR (neat): 3046, 1651, 1244, $1132 \mathrm{~cm}^{-1}$.

${ }^{1} \mathrm{H} \mathrm{NMR}\left(300 \mathrm{MHz}, \mathrm{CDCl}_{3}\right): \delta=5.43(\mathrm{~m}, 2 \mathrm{H}), 4.15-4.04(\mathrm{~m}, 2 \mathrm{H})$, 3.84-3.71 (m, 2 H), 1.89-1.82 (m, 2 H), 1.37 (s, 3 H), 1.50 (s, 3 H).

${ }^{13} \mathrm{C} \mathrm{NMR}\left(75 \mathrm{MHz}, \mathrm{CDCl}_{3}\right): \delta=137.4(\mathrm{C}), 103.1\left(\mathrm{CH}_{2}\right), 102.6(\mathrm{C})$, $61.3\left(2 \mathrm{CH}_{2}\right), 24.5(2 \mathrm{CH}), 20.5\left(2 \mathrm{CH}_{3}\right)$.

HRMS (ESI): $m / z[\mathrm{M}+\mathrm{Na}]^{+}$calcd for $\mathrm{C}_{9} \mathrm{H}_{14} \mathrm{NaO}_{2}: 177.0886$; found: 177.0880 .

[2-(Hydroxymethyl)-3-methylenecyclopropyl]methanol (1) A soln of compound $2(10.0 \mathrm{~g}, 64.9 \mathrm{mmol})$ in THF $(100 \mathrm{~mL})$ was cooled at $0{ }^{\circ} \mathrm{C}$, and aq $2 \mathrm{M} \mathrm{HCl} \operatorname{soln}(50 \mathrm{~mL})$ was added. The resulting soln was stirred for $2 \mathrm{~h}$ at r.t. The mixture was then cooled, neutralized with Amberlyst IRA-67, filtered, and concentrated under reduced pressure. Purification of the residue by column chromatography (silica gel) gave the meso-diol $1(6.01 \mathrm{~g}, 81 \%)$ as a colorless oil.

IR (neat): 3379, 3076, 1262, $1158 \mathrm{~cm}^{-1}$.

${ }^{1} \mathrm{H} \mathrm{NMR}\left(300 \mathrm{MHz}, \mathrm{CDCl}_{3}\right): \delta=5.36(\mathrm{~m}, 2 \mathrm{H}), 4.05-3.97(\mathrm{~m}, 2 \mathrm{H})$, $3.81(\mathrm{~m}, 2 \mathrm{H}), 3.42-3.27(\mathrm{~m}, 2 \mathrm{H}), 2.06-2.00(\mathrm{~m}, 2 \mathrm{H})$.

${ }^{13} \mathrm{C} \mathrm{NMR}\left(75 \mathrm{MHz}, \mathrm{CDCl}_{3}\right): \delta=135.3(\mathrm{C}), 105.1\left(\mathrm{CH}_{2}\right), 60.3(2$ $\left.\mathrm{CH}_{2}\right), 22.5(2 \mathrm{CH})$.

HRMS (ESI): $m / z[\mathrm{M}+\mathrm{Na}]^{+}$calcd for $\mathrm{C}_{6} \mathrm{H}_{10} \mathrm{NaO}_{2}: 137.0573$; found: 137.0573 .

(-)-(1R,2S)-2-(Hydroxymethyl)-3-methylenecyclopropylmethyl Acetate [(-)-4]

The meso-diol 1 (6.00 g, $52.6 \mathrm{mmol})$, PFL (600 mg), and vinyl acetate $(10 \mathrm{~mL})$ in $i-\mathrm{Pr}_{2} \mathrm{O}(50 \mathrm{~mL})$ were magnetically stirred at r.t. (TLC monitoring). After $1 \mathrm{~h}$, the mixture was filtered, the solvent was removed in vacuo and the residue was purified by column chromatography (silica gel) to give monoacetate (-)-4 (7.09 g, 86\%) as a colorless oil.

$[\alpha]_{\mathrm{D}}{ }^{25}-5.7\left(c 1.0, \mathrm{CHCl}_{3}\right)$.

IR (neat): 3381, 3061, 1748, 1259, $1152 \mathrm{~cm}^{-1}$.

${ }^{1} \mathrm{H}$ NMR $\left(300 \mathrm{MHz}, \mathrm{CDCl}_{3}\right): \delta=5.45(\mathrm{~s}, 2 \mathrm{H}), 4.45(\mathrm{dd}, J=12.4$, $5.1 \mathrm{~Hz}, 1 \mathrm{H}), 3.90(\mathrm{dd}, J=12.3,7.9 \mathrm{~Hz}, 1 \mathrm{H}), 3.88(\mathrm{~d}, J=12.4 \mathrm{~Hz}$, $1 \mathrm{H}), 3.45$ (dd, $J=12.3,9.6 \mathrm{~Hz}, 1 \mathrm{H}), 2.23$ (br s, $1 \mathrm{H}$ ), 2.12-2.05 (m, $1 \mathrm{H}$, partially overlapped), $2.09(\mathrm{~s}, 3 \mathrm{H}), 2.03-1.96(\mathrm{~m}, 1 \mathrm{H})$.

${ }^{13} \mathrm{C} \mathrm{NMR}\left(75 \mathrm{MHz}, \mathrm{CDCl}_{3}\right): \delta=171.2(\mathrm{C}), 133.5(\mathrm{C}), 105.6\left(\mathrm{CH}_{2}\right)$, $62.5\left(\mathrm{CH}_{2}\right), 60.6\left(\mathrm{CH}_{2}\right), 23.2(\mathrm{CH}), 21.2(\mathrm{CH}), 18.9\left(\mathrm{CH}_{3}\right)$.

HRMS (ESI): $m / z[\mathrm{M}+\mathrm{H}]^{+}$calcd for $\mathrm{C}_{8} \mathrm{H}_{13} \mathrm{O}_{3}: 157.0859$; found: 157.0857 .

\section{(-)-2-(Acetoxymethyl)-3-methylenecyclopropylmethyl 4,7,7- Trimethyl-3-oxo-2-oxabicyclo[2.2.1] heptane-1-carboxylate $[(-)-5]$}

To a soln of (-)-4 (100 mg, $0.64 \mathrm{mmol})$ and DMAP (11.7 mg, 0.1 $\mathrm{mmol})$ in pyridine $(15 \mathrm{~mL})$ at $5{ }^{\circ} \mathrm{C}$ under an argon atmosphere, was added (-)-(1S,4R)-camphanic acid chloride $(160 \mathrm{mg}, 0.70 \mathrm{mmol})$. The cooling bath was removed and the soln was stirred at r.t. (TLC monitoring) and the reaction was complete within $1 \mathrm{~h}$. The mixture was diluted with $\mathrm{CH}_{2} \mathrm{Cl}_{2}$ and then it was sequentially washed with $\mathrm{H}_{2} \mathrm{O}$, aq $1 \mathrm{M} \mathrm{HCl}$ soln, aq $1 \mathrm{M} \mathrm{NaHCO}_{3}$ soln, and brine. The organic layer was dried (anhyd $\mathrm{MgSO}_{4}$ ), filtered, and concentrated in vacuo. The crude mixture was subjected to column chromatography to afford (-)-5 (196 mg, 91\%) as a colorless oil.

$[\alpha]_{\mathrm{D}}^{25}-13.4\left(c 1.0, \mathrm{CHCl}_{3}\right)$. 
IR (neat): $3058,1741,1728,1174 \mathrm{~cm}^{-1}$.

${ }^{1} \mathrm{H}$ NMR $\left(300 \mathrm{MHz}, \mathrm{CDCl}_{3}\right): \delta=5.47(\mathrm{~s}, 2 \mathrm{H}), 4.42(\mathrm{dd}, J=11.7$, $6.8 \mathrm{~Hz}, 1 \mathrm{H}), 4.38(\mathrm{dd}, J=11.7,5.1 \mathrm{~Hz}, 1 \mathrm{H}), 4.10(\mathrm{dd}, J=11.7,8.2$ $\mathrm{Hz}, 1 \mathrm{H}), 3.91(\mathrm{dd}, J=11.7,8.6 \mathrm{~Hz}, 1 \mathrm{H}), 2.43$ (ddd, $J=12.1,8.6$, $4.6 \mathrm{~Hz}, 1 \mathrm{H}), 2.03(\mathrm{~s}, 3 \mathrm{H}), 2.06-1.90(\mathrm{~m}, 4 \mathrm{H}), 1.63(\mathrm{ddd}, J=12.3$, 9.3, 4.6 Hz, $1 \mathrm{H}), 1.07$ (s, $3 \mathrm{H}), 1.03$ (s, $3 \mathrm{H}), 0.94(\mathrm{~s}, 3 \mathrm{H})$.

${ }^{13} \mathrm{C} \mathrm{NMR}\left(75 \mathrm{MHz}, \mathrm{CDCl}_{3}\right): \delta=178.1(\mathrm{C}), 170.9(\mathrm{C}), 167.5(\mathrm{C})$, $132.8(\mathrm{C}), 106.3\left(\mathrm{CH}_{2}\right), 91.1(\mathrm{C}), 63.6\left(\mathrm{CH}_{2}\right), 62.0\left(\mathrm{CH}_{2}\right), 54.8(\mathrm{C})$, $54.2(\mathrm{C}), 30.7\left(\mathrm{CH}_{2}\right), 28.9\left(\mathrm{CH}_{2}\right), 21.0\left(\mathrm{CH}_{3}\right), 19.1(\mathrm{CH}), 18.6(\mathrm{CH})$, $16.8\left(2 \mathrm{CH}_{3}\right), 9.7\left(\mathrm{CH}_{3}\right)$.

HRMS (ESI): $m / z[\mathrm{M}+\mathrm{H}]^{+}$calcd for $\mathrm{C}_{18} \mathrm{H}_{25} \mathrm{O}_{6}: 337.1646$; found: 337.1646 .

(+)-2-(Hydroxymethyl)-3-methylenecyclopropylmethyl 4,7,7Trimethyl-3-oxo-2-oxabicyclo[2.2.1] heptane-1-carboxylate [(+)-6]

To a soln of (-)-5 (100 mg, $0.30 \mathrm{mmol})$ in $\mathrm{MeOH}(10 \mathrm{~mL})$ was added $\mathrm{K}_{2} \mathrm{CO}_{3}(50.0 \mathrm{mg}, 0.40 \mathrm{mmol})$. The mixture was stirred for $24 \mathrm{~h}$ at r.t., and then it was concentrated to remove $\mathrm{MeOH}$, diluted with $\mathrm{Et}_{2} \mathrm{O}$, filtered, and concentrated in vacuo. Purification of the residue by flash chromatography gave (+)-6 (76 $\mathrm{mg}, 86 \%)$ as a solid. Recrystallization in $\mathrm{Et}_{2} \mathrm{O}$-pentane gave $(+)-6$ as single crystals, suitable for X-ray diffraction; $\mathrm{mp} 81-83^{\circ} \mathrm{C}$.

$[\alpha]_{\mathrm{D}}^{25}+9.0\left(c 1.0, \mathrm{CHCl}_{3}\right)$.

IR (KBr): 3369, 3026, 1784, 1749, 1731, $1172 \mathrm{~cm}^{-1}$.

${ }^{1} \mathrm{H} \mathrm{NMR}\left(300 \mathrm{MHz}, \mathrm{CDCl}_{3}\right): \delta=5.44(\mathrm{~s}, 2 \mathrm{H}), 4.52(\mathrm{~m}, 1 \mathrm{H}), 4.08$ (dd, $J=11.8,9.1 \mathrm{~Hz}, 1 \mathrm{H}), 3.84($ br d, $J=13.5 \mathrm{~Hz}, 1 \mathrm{H}), 3.47(\mathrm{dd}$, $J=12.1,8.9 \mathrm{~Hz}, 1 \mathrm{H}), 2.49$ (br s, $1 \mathrm{H}), 2.32(\mathrm{dd}, J=8.9,4.6 \mathrm{~Hz}, 1$ H), 2.01-1.92 (m, 4 H), $1.62(\mathrm{ddd}, J=12.3,9.2,4.5 \mathrm{~Hz}, 1 \mathrm{H}), 1.11$ (s, $3 \mathrm{H}), 1.02(\mathrm{~s}, 3 \mathrm{H}), 0.93$ (s, $3 \mathrm{H})$.

${ }^{13} \mathrm{C}$ NMR $\left(75 \mathrm{MHz}, \mathrm{CDCl}_{3}\right): \delta=177.8(\mathrm{C}), 167.5(\mathrm{C}), 133.4(\mathrm{C})$, 105.6 $\left(\mathrm{CH}_{2}\right), 91.0(\mathrm{C}), 63.9\left(\mathrm{CH}_{2}\right), 60.3\left(\mathrm{CH}_{2}\right), 54.7(\mathrm{C}), 54.3(\mathrm{C})$, $30.7\left(\mathrm{CH}_{2}\right), 28.9\left(\mathrm{CH}_{2}\right), 22.9(\mathrm{CH}), 18.3(\mathrm{CH}), 16.7\left(2 \mathrm{CH}_{3}\right), 9.7$ $\left(\mathrm{CH}_{3}\right)$.

HRMS (ESI): $m / z[\mathrm{M}+\mathrm{H}]^{+}$calcd for $\mathrm{C}_{16} \mathrm{H}_{23} \mathrm{O}_{5}: 295.3547$; found: 295.3548.

2-(Acetoxymethyl)-3-methylenecyclopropylmethyl Acetate (3) To a stirred soln of meso-diol $1(1.00 \mathrm{~g}, 8.76 \mathrm{mmol})$ in pyridine (50 $\mathrm{mL})$ was added $\mathrm{Ac}_{2} \mathrm{O}(2.00 \mathrm{~mL}, 18.4 \mathrm{mmol})$ and the mixture was stirred for $24 \mathrm{~h}$ at r.t. The mixture was poured into $\mathrm{H}_{2} \mathrm{O}$ and extracted with EtOAc. The combined organic layers were washed with aq $10 \% \mathrm{CuSO}_{4}$ soln, $\mathrm{H}_{2} \mathrm{O}$, and brine, and then dried (anhyd $\mathrm{MgSO}_{4}$ ). Concentration of the organic layer in vacuo followed by purification of the residue by column chromatography (silica gel) gave 3 (1.58 g, 91\%) as a colorless oil.

IR (neat): 3039, 1779, 1261, $1171 \mathrm{~cm}^{-1}$.

${ }^{1} \mathrm{H}$ NMR $\left(300 \mathrm{MHz}, \mathrm{CDCl}_{3}\right): \delta=5.48(\mathrm{t}, J=2.2 \mathrm{~Hz}, 2 \mathrm{H}), 4.48(\mathrm{dd}$, $J=11.1,7.5 \mathrm{~Hz}, 2 \mathrm{H}), 3.95$ (dd, $J=11.1,8.5 \mathrm{~Hz}, 2 \mathrm{H}), 2.11$ (br s, 8 $\mathrm{H})$.

${ }^{13} \mathrm{C}$ NMR $\left(75 \mathrm{MHz}, \mathrm{CDCl}_{3}\right): \delta=170.7$ (2 C), $133.4(\mathrm{C}), 105.8$ $\left(\mathrm{CH}_{2}\right), 62.3\left(2 \mathrm{CH}_{2}\right), 20.9(2 \mathrm{CH}), 18.8\left(2 \mathrm{CH}_{3}\right)$.

HRMS (ESI): $m / z[\mathrm{M}+\mathrm{H}]^{+}$calcd for $\mathrm{C}_{10} \mathrm{H}_{15} \mathrm{O}_{4}: 199.2261$; found: 199.2263.

(+)-(1S,2R)-2-(Hydroxymethyl)-3-methylenecyclopropylmethyl Acetate $[(+)-4]$

To a soln of diacetate $3(1.00 \mathrm{~g}, 5.04 \mathrm{mmol})$ in $i-\mathrm{Pr}_{2} \mathrm{O}(50 \mathrm{~mL})$ and $\mathrm{BuOH}(10 \mathrm{~mL})$ was added PFL $(100.0 \mathrm{mg})$. The mixture was stirred at r.t. for $18 \mathrm{~h}$. Then, the mixture was filtered through a pad of Celite. Concentration of the organic layer in vacuo followed by purification by column chromatography (silica gel) gave (+)-4. IR, ${ }^{1} \mathrm{H}$ NMR, ${ }^{13} \mathrm{C}$ NMR, and HRMS data are identical with those described for $(-)-4 .[\alpha]_{\mathrm{D}}^{25}+5.9\left(c 1.0, \mathrm{CHCl}_{3}\right)$.

\section{(+)-(1R,2S)-2-(6-Chloropurin-9-ylmethyl)-3-methylenecyclo-} propylmethyl Acetate [(+)-7]

DIAD ( $379 \mu \mathrm{L}, 1.87 \mathrm{mmol})$ was added dropwise to a soln of $\mathrm{Ph}_{3} \mathrm{P}$ $(504 \mathrm{mg}, 1.87 \mathrm{mmol})$ in anhyd THF $(15 \mathrm{~mL})$ under an argon atmosphere at $0{ }^{\circ} \mathrm{C}$. The mixture was stirred for $30 \mathrm{~min}$ and then 6-chloropurine ( $297 \mathrm{mg}, 1.87 \mathrm{mmol}$ ) was added. The mixture was stirred for an additional $30 \mathrm{~min}$ and then a soln of alcohol (-)-4 (200 mg, $1.28 \mathrm{mmol})$ in anhyd THF $(15 \mathrm{~mL})$ was slowly added. The cooling bath was removed and the mixture was stirred at r.t. for $12 \mathrm{~h}$. The volatiles were evaporated in vacuo and the resulting residue was purified by column chromatography to give the carbonucleoside $(+)-7$ (322 $\mathrm{mg}, 86 \%$ ) as a foam.

$[\alpha]_{\mathrm{D}}^{25}+13.0\left(c 1.0, \mathrm{CHCl}_{3}\right)$.

IR (neat): 3029, 1758, 1241, $1136 \mathrm{~cm}^{-1}$.

${ }^{1} \mathrm{H}$ NMR $\left(300 \mathrm{MHz}, \mathrm{CDCl}_{3}\right): \delta=8.68(\mathrm{~s}, 1 \mathrm{H}), 8.23(\mathrm{~s}, 1 \mathrm{H}), 5.52$ $(\mathrm{t}, J=2.0 \mathrm{~Hz}, 1 \mathrm{H}), 5.43(\mathrm{t}, J=2.0 \mathrm{~Hz}, 1 \mathrm{H}), 4.50$ and $3.84(\mathrm{ABX}$, $J=12.3,10.2,5.1 \mathrm{~Hz}, 2 \mathrm{H}), 4.38$ and 4.28 (ABX, $J=14.7,8.5,6.8$ $\mathrm{Hz}, 2 \mathrm{H}), 2.36-2.25(\mathrm{~m}, 1 \mathrm{H}), 2.16-2.07(\mathrm{~m}, 1 \mathrm{H}), 1.91(\mathrm{~s}, 3 \mathrm{H})$.

${ }^{13} \mathrm{C}$ NMR $\left(75 \mathrm{MHz}, \mathrm{CDCl}_{3}\right): \delta=170.6(\mathrm{C}), 151.9(\mathrm{CH}), 151.8(\mathrm{C})$, $150.9(\mathrm{C}), 145.0(\mathrm{CH}), 132.2(\mathrm{C}), 131.5(\mathrm{C}), 106.9\left(\mathrm{CH}_{2}\right), 61.6$ $\left(\mathrm{CH}_{2}\right), 42.5\left(\mathrm{CH}_{2}\right), 20.8(\mathrm{CH}), 19.4(\mathrm{CH}), 19.2\left(\mathrm{CH}_{3}\right)$.

HRMS (ESI): $m / z[\mathrm{M}+\mathrm{H}]^{+}$calcd for $\mathrm{C}_{13} \mathrm{H}_{14} \mathrm{~N}_{4} \mathrm{O}_{2} \mathrm{Cl}$ : 293.0800; found: 293.0802 .

\section{(-)-(1R,2S)-[2-(6-Aminopurin-9-ylmethyl)-3-methylenecyclo-} propylmethyl]methanol [(-)-8]

A soln of $(+)-7(100 \mathrm{mg}, 0.34 \mathrm{mmol})$ in sat. methanolic $\mathrm{NH}_{3}(20$ $\mathrm{mL}$ ) was heated at $100^{\circ} \mathrm{C}$ for $10 \mathrm{~h}$ in a Parr stainless steel sealed reaction vessel. Then, the reaction mixture was allowed to cool to r.t. and concentrated in vacuo. The residue was purified by column chromatography to give carbonucleoside $(-)-8(72.3 \mathrm{mg}, 92 \%)$ as a white solid; mp $170-172^{\circ} \mathrm{C}$.

$[\alpha]_{\mathrm{D}}{ }^{25}-35.4(\mathrm{c} 1.0, \mathrm{MeOH})$.

IR (KBr): 3387, 3031, 1603, 1274, $1181 \mathrm{~cm}^{-1}$.

${ }^{1} \mathrm{H}$ NMR $\left(300 \mathrm{MHz}\right.$, DMSO- $\left.d_{6}\right): \delta=8.22(\mathrm{~s}, 1 \mathrm{H}), 8.17(\mathrm{~s}, 1 \mathrm{H}), 7.28$ (s, $2 \mathrm{H}), 5.41(\mathrm{~s}, 1 \mathrm{H}), 5.32(\mathrm{~s}, 1 \mathrm{H}), 5.04(\mathrm{t}, J=5.3 \mathrm{~Hz}, 1 \mathrm{H}), 4.30$ and 4.21 (ABX, $J=14.7,8.1,6.4 \mathrm{~Hz}, 2 \mathrm{H}), 3.78(\mathrm{dt}, J=11.3,5.4$ $\mathrm{Hz}, 1 \mathrm{H}), 3.46$ (m, $1 \mathrm{H}$, partially overlapped), 2.16 (m, $1 \mathrm{H}), 1.97$ $(\mathrm{m}, 1 \mathrm{H})$.

${ }^{13} \mathrm{C}$ NMR (75 MHz, DMSO- $\left.d_{6}\right): \delta=156.0(\mathrm{C}), 152.3(\mathrm{CH}), 149.4$ (C), $140.9(\mathrm{CH}), 135.3(\mathrm{C}), 118.7(\mathrm{C}), 104.4\left(\mathrm{CH}_{2}\right), 58.5\left(\mathrm{CH}_{2}\right)$, $40.8\left(\mathrm{CH}_{2}\right), 22.8(\mathrm{CH}), 19.3(\mathrm{CH})$.

HRMS (ESI): $m / z[\mathrm{M}+\mathrm{H}]^{+}$calcd for $\mathrm{C}_{11} \mathrm{H}_{14} \mathrm{~N}_{5} \mathrm{O}: 232.2645$; found: 232.2644 .

\section{(-)-(1R,2S)-\{2-[6-(Cyclopropylamino)purin-9-ylmethyl]-3- methylenecyclopropylmethyl\} methanol [(-)-9]}

A soln of $(+)-7(65.0 \mathrm{mg}, 0.22 \mathrm{mmol})$ and cyclopropylamine $(1.0$ $\mathrm{mL})$ in $\mathrm{MeOH}(5.0 \mathrm{~mL})$ was stirred at r.t. for $12 \mathrm{~h}$ under argon. The reaction mixture was allowed to cool to r.t. and concentrated in vacuo. Then, the resulting residue was dissolved into sat. methanolic $\mathrm{NH}_{3}(15 \mathrm{~mL})$ in a flask fitted with a rubber stopper at r.t. for $5 \mathrm{~h}$. After evaporation of the solvent in vacuo, the residue was purified by column chromatography to give (-)-9 $(53.6 \mathrm{mg}, 89 \%)$ as a white solid; mp $184-185^{\circ} \mathrm{C}$.

$[\alpha]_{\mathrm{D}}^{25}-26.4(c 1.0, \mathrm{MeOH})$.

IR (KBr): 3406, 3017, 1598, 1249, $1173 \mathrm{~cm}^{-1}$.

${ }^{1} \mathrm{H} \mathrm{NMR}\left(300 \mathrm{MHz}, \mathrm{MeOH}-d_{4}\right): \delta=8.26(\mathrm{~s}, 1 \mathrm{H}), 8.19(\mathrm{~s}, 1 \mathrm{H}), 5.46$ $(\mathrm{t}, J=2.0 \mathrm{~Hz}, 1 \mathrm{H}), 5.37(\mathrm{t}, J=2.0 \mathrm{~Hz}, 1 \mathrm{H}), 4.37$ and $4.23(\mathrm{ABX}$, $J=14.6,8.2,5.9 \mathrm{~Hz}, 2 \mathrm{H}), 3.93(\mathrm{dd}, J=12.1,5.2 \mathrm{~Hz}, 1 \mathrm{H}), 3.57$ (dd, $J=12.1,9.3 \mathrm{~Hz}, 1 \mathrm{H}), 2.97-2.90(\mathrm{~m}, 1 \mathrm{H}), 2.23-2.12(\mathrm{~m}, 1 \mathrm{H})$, 2.11-2.01 (m, $1 \mathrm{H}), 0.90-0.82$ (m, $2 \mathrm{H}), 0.66-0.59$ (m, $2 \mathrm{H})$. 
${ }^{13} \mathrm{C}$ NMR $\left(75 \mathrm{MHz}, \mathrm{MeOH}-d_{4}\right): \delta=157.0(\mathrm{C}), 153.4(\mathrm{CH}), 149.6$ (C), $142.4(\mathrm{CH}), 135.5(\mathrm{C}), 120.4(\mathrm{C}), 105.4\left(\mathrm{CH}_{2}\right), 60.2\left(\mathrm{CH}_{2}\right)$, $42.5\left(\mathrm{CH}_{2}\right), 24.6(\mathrm{CH}), 24.1(\mathrm{CH}), 20.7(\mathrm{CH}), 7.6\left(2 \mathrm{CH}_{2}\right)$.

HRMS (ESI): $m / z[\mathrm{M}+\mathrm{H}]^{+}$calcd for $\mathrm{C}_{14} \mathrm{H}_{18} \mathrm{ON}_{5}: 272.1506$; found: 272.1505 .

\section{(-)-(1S,2R)-2-(Acetoxymethyl)-3-methylenecyclopropanecar-} boxylic Acid [(-)-10]

A soln of Jones reagent [prepared by mixing $\mathrm{CrO}_{3}(26.7 \mathrm{~g})$ with concd $\mathrm{H}_{2} \mathrm{SO}_{4}(23 \mathrm{~mL})$, followed by dilution with $\mathrm{H}_{2} \mathrm{O}$ to a final volume of $100 \mathrm{~mL}$ ] was added to a soln of alcohol (-)-4 (4.00 g, 25.6 $\mathrm{mmol})$ in acetone $(100 \mathrm{~mL})$. Addition of Jones reagent $(\mathrm{ca} .20 \mathrm{~mL})$ was continued at $0{ }^{\circ} \mathrm{C}$ until the reddish color persisted for at least 5 $\min$. The resulting soln was stirred for a further $1 \mathrm{~h}$ at r.t., and the excess oxidizing reagent was quenched with $i$-PrOH. The resulting soln was then diluted with $\mathrm{H}_{2} \mathrm{O}$ followed by repeated extraction with EtOAc. The combined organic phases were washed with $10 \%$ aq $\mathrm{NaOH}$ soln and the aqueous extracts were acidified to $\mathrm{pH}<2$ with aq $6 \mathrm{M} \mathrm{HCl}$ soln. The resulting soln was extracted with EtOAc. The organic phases were combined, dried, and concentrated in vacuo to give acid (-)-10 (3.96 g, 91\%) as a colorless oil.

$[\alpha]_{\mathrm{D}}^{25}-19.3\left(\mathrm{c} 1.0, \mathrm{CHCl}_{3}\right)$.

IR (neat): 3252, 3061, 1736, 1710, 1249, $1150 \mathrm{~cm}^{-1}$.

${ }^{1} \mathrm{H}$ NMR $\left(300 \mathrm{MHz}, \mathrm{CDCl}_{3}\right): \delta=10.3($ br s, $1 \mathrm{H}), 5.63$ and 5.61 $(\mathrm{AB}, J=2.1 \mathrm{~Hz}, 2 \mathrm{H}), 4.52(\mathrm{~m}, 1 \mathrm{H}), 4.22(\mathrm{~m}, 1 \mathrm{H}), 2.51-2.49(\mathrm{~m}$, $2 \mathrm{H}), 2.12(\mathrm{~s}, 3 \mathrm{H})$.

${ }^{13} \mathrm{C}$ NMR $\left(75 \mathrm{MHz}, \mathrm{CDCl}_{3}\right): \delta=176.4(\mathrm{C}), 171.1(\mathrm{C}), 130.5(\mathrm{C})$, $106.8\left(\mathrm{CH}_{2}\right), 60.8\left(\mathrm{CH}_{2}\right), 23.2(\mathrm{CH}), 21.7(\mathrm{CH}), 20.8\left(\mathrm{CH}_{3}\right)$.

HRMS (ESI): $m / z[\mathrm{M}+\mathrm{H}]^{+}$calcd for $\mathrm{C}_{8} \mathrm{H}_{11} \mathrm{O}_{4}: 171.0651$; found: 171.0653 .

\section{(+)-(1R,2S)-2-(Azidocarbonyl)-3-methylenecyclopropylmethyl} Acetate [(+)-11]

A soln of compound (-)-10 (500 mg, $2.93 \mathrm{mmol})$ was cooled at $0{ }^{\circ} \mathrm{C}$, and oxalyl chloride $(1.00 \mathrm{~g}, 5.86 \mathrm{mmol})$ was added. The soln was allowed to warm to r.t. and stirred for $1 \mathrm{~h}$. The soln was concentrated at reduced pressure and the resulting residue was taken up in acetone $(16 \mathrm{~mL})$ and transferred dropwise to a vigorously stirred soln of $\mathrm{NaN}_{3}(773.0 \mathrm{mg}, 11.8 \mathrm{mmol})$ in $\mathrm{H}_{2} \mathrm{O}(35 \mathrm{~mL})$ at $0{ }^{\circ} \mathrm{C}$. The resulting soln was maintained at $0{ }^{\circ} \mathrm{C}$ for $15 \mathrm{~min}$, at which time it was partitioned between EtOAc and $\mathrm{H}_{2} \mathrm{O}$. After separation, the aqueous layer was extracted with EtOAc and the combined organic layers were washed with $\mathrm{H}_{2} \mathrm{O}$ and brine, and then dried (anhyd $\left.\mathrm{MgSO}_{4}\right)$ and concentrated in vacuo to provide acyl azide (+)-11 (453.0 $\mathrm{mg}, 79 \%$ ) as a colorless oil.

$[\alpha]_{\mathrm{D}}^{25}+29.0\left(c 1.0, \mathrm{CHCl}_{3}\right)$.

IR (neat): 3064, 2132, 1749, 1238, $1157 \mathrm{~cm}^{-1}$.

${ }^{1} \mathrm{H} \mathrm{NMR}\left(300 \mathrm{MHz}, \mathrm{CDCl}_{3}\right): \delta=5.60$ and $5.58(\mathrm{AB}, J=2.4 \mathrm{~Hz}, 2$ H), $4.53(\mathrm{~m}, 1 \mathrm{H}), 4.16(\mathrm{~m}, 1 \mathrm{H}), 2.55-2.41(\mathrm{~m}, 2 \mathrm{H}), 2.04(\mathrm{~s}, 3 \mathrm{H})$.

${ }^{13} \mathrm{C} \mathrm{NMR}\left(75 \mathrm{MHz}, \mathrm{CDCl}_{3}\right): \delta=176.7(\mathrm{C}), 170.8(\mathrm{C}), 130.8(\mathrm{C})$, $107.3\left(\mathrm{CH}_{2}\right), 60.3\left(\mathrm{CH}_{2}\right), 24.8(\mathrm{CH}), 24.1(\mathrm{CH}), 20.8\left(\mathrm{CH}_{3}\right)$.

\section{(+)-(1R,2S)-2-(tert-Butoxycarbonylamino)-3-methylenecyclo-} propylmethyl Acetate $[(+)-13]$

A soln of acyl azide $(+)-11(400 \mathrm{mg}, 2.04 \mathrm{mmol})$ in benzene (20 $\mathrm{mL}$ ) was heated at $75^{\circ} \mathrm{C}$ until the evolution of gas ceased $(\sim 3 \mathrm{~h})$, and the mixture was cooled to r.t. The resulting isocyanate was dissolved in anhyd $t$-BuOH $(2.0 \mathrm{~mL})$ and the soln was refluxed for $4 \mathrm{~h}$. The mixture was cooled to r.t. and the solvent was removed in vacuo. The residue was purified by flash chromatography to give carbamate (+)-13 (395 $\mathrm{mg}, 75 \%)$ as a colorless oil.

$[\alpha]_{\mathrm{D}}{ }^{25}+41.4\left(\mathrm{c} 1.0, \mathrm{CHCl}_{3}\right)$.

IR (neat): $3340,3053,1759,1717,1143 \mathrm{~cm}^{-1}$.

${ }^{1} \mathrm{H}$ NMR $\left(300 \mathrm{MHz}, \mathrm{CDCl}_{3}\right): \delta=5.72(\mathrm{~s}, 1 \mathrm{H}), 5.55(\mathrm{~s}, 1 \mathrm{H}), 4.89$ (br d, $J=5.9 \mathrm{~Hz}, 1 \mathrm{H}$ ), 4.18 and $4.03(\mathrm{ABX}, J=10.7,10.3,5.5 \mathrm{~Hz}$, $2 \mathrm{H}), 3.35$ (br s, $1 \mathrm{H}), 2.11-2.07$ (m, $1 \mathrm{H}), 2.05(\mathrm{~s}, 3 \mathrm{H}), 1.44(\mathrm{~s}, 9 \mathrm{H})$.
${ }^{13} \mathrm{C}$ NMR (75 MHz, $\mathrm{CDCl}_{3}$ ): $\delta=171.1(\mathrm{C}), 156.3(\mathrm{C}), 132.5(\mathrm{C})$, $108.3\left(\mathrm{CH}_{2}\right), 79.7(\mathrm{C}), 61.0\left(\mathrm{CH}_{2}\right), 29.1(\mathrm{CH}), 27.8\left(3 \mathrm{CH}_{3}\right), 21.3$ (CH), $21.1\left(\mathrm{CH}_{3}\right)$.

HRMS (ESI): $m / z[\mathrm{M}+\mathrm{H}]^{+}$calcd for $\mathrm{C}_{12} \mathrm{H}_{20} \mathrm{O}_{3} \mathrm{~N}$ : 226.2965; found: 226.2970 .

\section{[2-(Hydromethyl)-3-methylenecyclopropylmethyl]ammonium Chloride (14)}

A soln of (+)-13 (200.0 mg, $0.77 \mathrm{mmol})$ in aq $1.2 \mathrm{M} \mathrm{HCl}$ soln-glacial AcOH $(10 \mathrm{~mL}(1: 1.5)$ was stirred at r.t. for $12 \mathrm{~h}$. After removal of the volatiles, the residue was triturated with $\mathrm{Et}_{2} \mathrm{O}$. After filtration, the solid residue was placed under vacuum and gave product $\mathbf{1 4}$ $(89.6 \mathrm{mg}, 85 \%)$ as a brown oil. The chlorohydrate salt 14 was sufficiently pure to be used in the next step without purification.

IR (neat): 3386, 3296, 3058, 1247, $1172 \mathrm{~cm}^{-1}$.

${ }^{1} \mathrm{H} \mathrm{NMR}\left(300 \mathrm{MHz}, \mathrm{MeOH}-d_{4}\right): \delta=4.32($ br s, $1 \mathrm{H}), 4.17(\mathrm{t}, J=2.0$ $\mathrm{Hz}, 1 \mathrm{H}), 2.41(\mathrm{dd}, J=12.0,4.7 \mathrm{~Hz}, 1 \mathrm{H}), 2.16(\mathrm{dd}, J=12.0,7.0 \mathrm{~Hz}$, $1 \mathrm{H}), 1.76(\mathrm{~m}, 1 \mathrm{H}), 0.68-0.61(\mathrm{~m}, 1 \mathrm{H})$.

${ }^{13} \mathrm{C}$ NMR $\left(75 \mathrm{MHz}, \mathrm{MeOH}-d_{4}\right): \delta=129.9(\mathrm{C}), 110.7\left(\mathrm{CH}_{2}\right), 59.4$ $\left(\mathrm{CH}_{2}\right), 28.3(\mathrm{CH}), 23.4(\mathrm{CH})$.

HRMS (ESI): $m / z[M]^{+}$calcd for $\mathrm{C}_{5} \mathrm{H}_{10} \mathrm{NO}$ : 100.0757; found: 100.0753 .

(+)-(1S,2S)-1-[2-(Hydromethyl)-3-methylenecyclopropyl]-1Hpyrimidine-2,4-dione $[(+)-16]$

To a soln of chlorocarbonyl isocyanate $(75.0 \mu \mathrm{L}, 0.88 \mathrm{mmol})$ in THF $(2.0 \mathrm{~mL})$ was slowly added a soln of ethyl vinyl ether $(110 \mu \mathrm{L}$, $1.04 \mathrm{mmol})$ in THF $(2.0 \mathrm{~mL})$ at $0{ }^{\circ} \mathrm{C}$. The resulting soln was stirred for $20 \mathrm{~min}$ at $0{ }^{\circ} \mathrm{C}$ and $\mathrm{Et}_{3} \mathrm{~N}(130 \mu \mathrm{L}, 0.88 \mathrm{mmol})$ in THF $(2.0 \mathrm{~mL})$ was then slowly added. The resulting suspension was stirred for 5 min at $0{ }^{\circ} \mathrm{C}$ then cooled down to $-40^{\circ} \mathrm{C}$. A soln of chlorohydrate salt $14(100 \mathrm{mg}, 0.74 \mathrm{mmol})$ in DMF $(2.0 \mathrm{~mL})$ was rapidly added to the mixture and the suspension was allowed to slowly warm up to r.t. Then, volatiles were evaporated and the residue was dissolved in dioxane $(5.0 \mathrm{~mL})$. To this soln was added aq $0.5 \mathrm{M} \mathrm{H}_{2} \mathrm{SO}_{4}$ soln $(9.0$ $\mathrm{mL}$ ) and the mixture was heated at $80^{\circ} \mathrm{C}$ for $2 \mathrm{~h}$. Then the soln was cooled to r.t. and basified with aq $3 \mathrm{M} \mathrm{NH}_{3}$ soln $(7.0 \mathrm{~mL})$. The ammonium salts were precipitated with $\mathrm{MeOH}$ and the suspension was filtered. The filtrate was evaporated to dryness and the crude material was purified by column chromatography to afford $(+)-\mathbf{1 6}(107$ $\mathrm{mg}, 75 \%$ ) as a white solid; $\mathrm{mp} 183-184^{\circ} \mathrm{C}$.

$[\alpha]_{\mathrm{D}}^{25}+10.0(c 1.0, \mathrm{MeOH})$.

IR (KBr): 3305, 3074, 1685, 1254, $1146 \mathrm{~cm}^{-1}$.

${ }^{1} \mathrm{H}$ NMR $\left(300 \mathrm{MHz}\right.$, DMSO- $\left.d_{6}\right): \delta=11.3(\mathrm{~s}, 1 \mathrm{H}), 7.51(\mathrm{~d}, J=7.9$ $\mathrm{Hz}, 1 \mathrm{H}), 6.02(\mathrm{~s}, 1 \mathrm{H}), 5.80(\mathrm{~s}, 1 \mathrm{H}), 5.51(\mathrm{~d}, J=7.9 \mathrm{~Hz}, 1 \mathrm{H}), 4.56$ $(\mathrm{d}, J=5.6 \mathrm{~Hz}, 1 \mathrm{H}), 3.82(\mathrm{~d}, J=7.8 \mathrm{~Hz}, 1 \mathrm{H}), 3.39-3.18(\mathrm{~m}, 2 \mathrm{H}$, partially overlapped with DOH), 2.25-2.10 (m, $1 \mathrm{H})$.

${ }^{13} \mathrm{C}$ NMR (75 MHz, DMSO- $d_{6}$ ): $\delta=163.0(\mathrm{C}), 151.4(\mathrm{C}), 144.1$ $(\mathrm{CH}), 130.0(\mathrm{C}), 110.3\left(\mathrm{CH}_{2}\right), 99.8(\mathrm{CH}), 57.3\left(\mathrm{CH}_{2}\right), 33.7(\mathrm{CH})$, $24.3(\mathrm{CH})$.

HRMS (ESI): $m / z[\mathrm{M}+\mathrm{H}]^{+}$calcd for $\mathrm{C}_{9} \mathrm{H}_{11} \mathrm{O}_{3} \mathrm{~N}_{2}: 195.0760$; found: 195.0760 .

\section{Acknowledgment}

G.O. thanks the government of Gabon for a grant. The authors are grateful to Amano Pharmaceuticals for the kind gift of PFL. The authors are also thankful to Dr. N. Vanthuyne for HPLC analyses, to Dr. M. Giorgi for X-ray diffraction analyses and to CNRS and AixMarseille Université for their financial support. P. Fournier is gratefully acknowledged for the English revision of the manuscript.

Supporting Information for this article is available online at http://www.thieme-connect.com/ejournals/toc/synlett. 


\section{References}

(1) (a) De Clercq, E. Antiviral Res. 2005, 67, 56. (b) De Clercq, E. Curr. Opin. Microbiol. 2005, 8, 552. (c) Mathé, C.; Gosselin, G. Antiviral Res. 2006, 71, 276. (d) Field, H. J.; De Clercq, E. Antiviral Chem. Chemother. 2008, 19, 51. (e) De Clercq, E.; Field, H. J. Antiviral Chem. Chemother. 2008, 19,63 .

(2) (a) Vince, R.; Hua, M.; Brownell, J.; Daluge, S.; Lee, F.; Shannon, W. M.; Lavelle, G. C.; Qualls, J.; Weislow, O. S.; Kiser, R.; Canonico, P. G.; Schultz, R. H.; Narayanan, V. L.; Mayo, J. G.; Shoemaker, R. H.; Boyd, M. R. Biochem. Biophys. Res. Commun. 1988, 156, 1046. (b) Vince, R.; Hua, M. J. Med. Chem. 1990, 33, 17.

(3) (a) Daluge, S. M. US 5034394, 1991. (b) Good, S. S.; Daluge, S. M.; Ching, S. V.; Ayers, K. M.; Mahony, W. B.; Faletto, M. B.; Domin, B. A.; Owens, B. S.; Dornsife, R. E.; McDowell, J. A.; Lafon, S. W.; Symonds, W. T. Antiviral Res. 1995, 26, A229.

(4) For some representative examples, see: (a) Rao, J. R.; Schinazi, R. F.; Chu, C. K. Bioorg. Med. Chem. 2007, 15, 839. (b) Liu, L. J.; Ko, O. H.; Hong, J. H. Bull. Korean Chem. Soc. 2008, 29, 1723. (c) Abeijón, P.; Blanco, J. M.; Caamaño, O.; Fernández, F.; García, M. D.; García-Mera, X.; Rodríguez-Borges, J. E.; Balzarini, J.; De Clercq, E. Synthesis 2009, 2766. (d) Ugliarolo, E. A.; Lantaño, B.; Moltrasio, G. Y.; Moglioni, A. G. Tetrahedron: Asymmetry 2009, 20, 1848.

(5) For reviews, see: (a) Ferrero, M.; Gotor, V. Chem. Rev. 2000, 100, 4319. (b) Agrofoglio, L. A.; Gillaizeau, I.; Saito, Y. Chem. Rev. 2003, 103, 1875. (c) Rodriguez, J. B.; Comin, M. J. Mini-Rev. Med. Chem. 2003, 3, 95. For a recent book, see: (d) Modified Nucleosides in Biochemistry, Biotechnology and Medicine; Herdewijn, P., Ed.; WileyVCH: Weinheim, 2008.

(6) Bisacchi, G. S.; Chao, S. T.; Bachard, C.; Daris, J. P.; Innaimo, S.; Jacobs, G. A.; Kocy, O.; Lapointe, P.; Martel, A.; Merchant, Z.; Slusarchyk, W. A.; Sundeen, J. E.; Young, M. G.; Colonno, R.; Zahler, R. Bioorg. Med. Chem. Lett. 1997, 7, 127.

(7) For a review: Zemlicka, J. Adv. Antiviral Drug Des. 2007, 5, 113.

(8) (a) Qiu, Y.-L.; Ksebati, M. B.; Ptak, R. G.; Fan, B. Y.; Breitenbach, J. M.; Lin, J.-S.; Cheng, Y.-C.; Kern, E. R.; Drach, J. C.; Zemlicka, J. J. Med. Chem. 1998, 41, 10. (b) Qiu, Y.-L.; Ptak, R. G.; Fan, B. Y.; Breitenbach, J. M.; Lin, J.-S.; Cheng, Y.-C.; Kern, E. R.; Drach, J. C.; Zemlicka, J. Antiviral Chem. Chemother. 1998, 9, 341. (c) Qiu, Y.-L.; Zemlicka, J. Angew. Chem. Int. Ed. 1998, 37, 1441.

(d) Guan, H.-P.; Ksebati, M. B.; Cheng, Y.-C.; Drach, J. C.; Kern, E. R.; Zemlicka, J. J. Org. Chem. 2000, 65, 1280. (e) Wang, R.; Ksebati, M. B.; Corbett, T. H.; Earl, R. K.; Drach, J. C.; Zemlicka, J. J. Med. Chem. 2001, 44, 4019.

(9) (a) Zhou, S.; Breitenbach, J. M.; Borysko, K. Z.; Drach, J. C.; Kern, E. R.; Gullen, E.; Cheng, Y.-C.; Zemlicka, J. J. Med. Chem. 2004, 47, 566. (b) Kushner, N. L.; WilliamsAziz, S. L.; Hartline, C. B.; Harden, E. A.; Zhou, S.; Yan, Z.; Zemlicka, J.; Kern, E. R. Antiviral Res. 2004, 62, A68. (c) Zhaohua, Y.; Kern, E. R.; Gullen, E.; Cheng, Y.-C.; Drach, J. C.; Zemlicka, J. J. Med. Chem. 2005, 48, 91.

(10) Zemlicka, J. Adv. Antiviral Drug Des. 2007, 5, 113.

(11) Obame, G.; Pellissier, H.; Vanthuyne, N.; Bongui, J.-B.; Audran, G. Tetrahedron Lett. 2011, 52, 1082.

(12) Elliott, W. J.; Fried, J. J. Org. Chem. 1976, 41, 2469.

(13) (a) Binger, P.; Arora, S. Synthesis 1974, 801. (b) Brandi, A.; Goti, A. Chem. Rev. 1998, 98, 589. (c) de Meijere, A.; Becker, H.; Stolle, A.; Kozhushkov, S. I.; Bes, M. T.; Salaün, J.; Noltemeyer, M. Chem. Eur. J. 2005, 11, 2471.
(14) (a) Gerlach, H. Helv. Chim. Acta 1985, 68, 1815.

(b) Gerlach, H.; Kappes, D.; Boekman, K. Jr.; Maw, G. N. Org. Synth. 1993, 71, 48.

(15) CCDC-785043 contains the supplementary crystallographic data for this paper. These data can be obtained free of charge at www.ccdc.cam.ac.uk/conts/retrieving.html [or from the Cambridge Crystallographic Data Centre, 12, Union Road, Cambridge CB2 1EZ, UK; fax: +44(1223)336033; e-mail: deposit@ccdc.cam.ac.uk].

(16) (a) Mitsunobu, O. Synthesis 1981, 1. (b) Jenny, T. F.; Previsani, N.; Benner, S. A. Tetrahedron Lett. 1991, 32, 7029. (c) Wachtmeister, J.; Classon, B.; Samuelsson, B. Tetrahedron 1995, 51, 2029. (d) Crimmins, M. T. Tetrahedron 1998, 54, 9229.

(17) (a) Curtius, T. Chem. Ztg. 1912, 35, 249. (b) Tanaka, M.; Norimine, Y.; Fujita, T.; Suemune, H. J. Org. Chem. 1996, 61, 6952. (c) Aggarwal, V. K.; de Vicente, V.; Bonnert, R. V. Org. Lett. 2001, 3, 2785. (d) Banthorpe, D. V. Rearrangements Involving Azido Groups, In The Chemistry of the Azido Group; Patai, S., Ed.; Wiley: New York, 1971, 397-340.

(18) (a) Bowden, K.; Heilbron, I. M.; Jones, E. R. H.; Weedon, D. C. L. J. Chem. Soc. 1946, 39. (b) Luzzio, F. A.; Guziec, F. S. Jr. Org. Prep. Proced. Int. 1988, 20, 533. (c) Luzzio, F. A. Org. React. 1998, 53, 1. (d) Li, D.; Agnihotri, G.; Dakoji, S.; Oh, E.; Lantz, M.; Liu, H.-w. J. Am. Chem. Soc. 1999, 121, 9034.

(19) (a) Kazuta, Y.; Hirano, K.; Natsume, K.; Yamada, S.; Kimura, R.; Matsumoto, S.-I.; Furuichi, K.; Matsuda, A.; Shuto, S. J. Med. Chem. 2003, 46, 1980. (b) Kedrowski, B. L. J. Org. Chem. 2003, 68, 5403.

(20) (a) Weinstock, J. J. Org. Chem. 1961, 26, 3511. (b) Jaafar, I.; Francis, G.; Danion-Bougot, R.; Danion, D. Synthesis 1993, 56. (c) Vallgårda, J.; Appelberg, U.; Arvidsson, L.-E.; Hjorth, S.; Svensson, B. E.; Hacksell, U. J. Med. Chem. 1996, 39, 1485.

(21) Neutral conditions: (a) Murata, K.; Wang, S.-Z.; Morizawa, Y.; Oharu, K. J. Fluorine Chem. 2006, 127, 1125. Acidic conditions: (b) Cho, T. P.; Long, Y. F.; Gang, L. Z.; Yang, W.; Jun, L. H.; Yuan, S. G.; Hong, F. J.; Lin, W.; Liang, G. D.; Lei, Z.; Jing, L. J.; Shen, G. A.; Hong, S. G.; Dan, W.; Ying, F.; Ke, Y. P.; Ying, L.; Jun, F.; Tai, M. X. Bioorg. Med. Chem. Lett. 2010, 20, 3565. (c) Johnson, D. J.; Forbes, I. T.; Watson, S. P.; Garzya, V.; Stevenson, G. I.; Walker, G. R.; Mudhar, H. S.; Flynn, S. T.; Wyman, P. A.; Smith, P. W.; Murkitt, G. S.; Lucas, A. J.; Mookherjee, C. R.; Watson, J. M.; Gartlon, J. E.; Bradford, A. M.; Brown, F. Bioorg. Med. Chem. Lett. 2010, 20, 5434. Basic conditions:

(d) Neufellner, E.; Kapeller, H.; Griengl, H. Tetrahedron 1998, 54, 11043.

(22) Gajcy, K.; Pękala, J.; Frąckowiak-Wojtasek, B.; Librowski, T.; Lochynński, S. Tetrahedron: Asymmetry 2010, 21, 2015.

(23) (a) Zhao, Y.; Yang, T.; Lee, M.; Lee, D.; Newton, M. G.; Chu, C. K. J. Org. Chem. 1995, 60, 5236. (b) Yoshida, S. Y.; Meyer, O. G. J.; Rosen, T. C.; Haufe, G.; Ye, S.; Sloan, M. J.; Kirk, K. L. J. Med. Chem. 2004, 47, 1796.

(24) (a) Montgomery, J. A.; Temple, C. Jr. J. Am. Chem. Soc. 1957, 79, 5238. (b) Shealy, Y. F.; Clayton, J. D. J. Am. Chem. Soc. 1966, 88, 3885. (c) Shealy, Y. F.; Clayton, J. D. J. Am. Chem. Soc. 1969, 91, 3075. (d) Arita, M.; Adachi, K.; Ito, Y.; Sawai, H.; Ohno, M. J. Am. Chem. Soc. 1983, 105, 4049.

(25) (a) Shealy, Y. F.; O'Dell, C. A. J. Heterocycl. Chem. 1976, 13, 1015. (b) Lin, T.-S.; Zhang, X.-H.; Wang, Z.-H.; Prusoff, W. H. J. Med. Chem. 1988, 31, 484. (c) Gosselin, G.; Griffe, L.; Meillon, J.-C.; Storer, R. Tetrahedron 2006, 62, 906. 